\title{
The immunological implication of the new vitamin D metabolism
}

\author{
GIULIA BIVONA ${ }^{l}$, LUISA AGNELLO ${ }^{2}$, MARCELLO CIACCIO ${ }^{1,2}$
}

${ }^{1}$ Section of Clinical Biochemistry and Clinical Molecular Medicine, Department of Biopathology and Medical Biotechnologies, University of Palermo, Palermo, Italy

${ }^{2}$ Department and U.O.C. Laboratory Medicine, University Hospital "Paolo Giaccone" of Palermo, Palermo, Italy

\begin{abstract}
Vitamin D is a neuro-hormone regulating calcium-phosphate homeostasis, cell proliferation, and immunomodulation. Exogenous and endogenous vitamin $D$ is inactive, and two hydroxylations are required to produce the active hormone. The first hydroxylation is unique to the liver, while the second step occurs in kidney, brain, lung, prostate, placenta, and immune cells. Kidney-derived calcitriol regulates calcium homeostasis. Active hormone produced by brain and immune cells mediates immune system response; lung calcitriol is involved in fighting respiratory tract infections; finally, prostate and placenta vitamin $D$ regulates cells growth and proliferation within such tissues. Immune modulation by vitamin D includes enhancing innate immune response, attenuating and stimulating Th1 and Th2 cell proliferation, respectively, and promoting self-tolerance. Hypovitaminosis $D$ is a common finding in several autoimmune diseases. It is unclear whether hypovitaminosis D could be a consequence or a cause of autoimmune diseases and whether vitamin D supplementation has an impact on these patients. Moreover, there is no consensus on oral cholecalciferol dosage for supplementation. More interventional studies are required to better define how vitamin $D$ could represent both a causation agent in autoimmunity and a target for therapeutic strategies in autoimmune patients.
\end{abstract}

Key words: vitamin D, CYPs, VDR, immunomodulation, autoimmunity.

(Centr Eur J Immunol 2018; 43 (3): 331-334)

\section{Introduction}

Vitamin D refers to two compounds, vitamin D2 and vitamin D3. Vitamin D2 derives from irradiation of the plant sterol, ergosterol, and is provided by the diet. Vitamin D3 originates from a cutaneous compound by endogenous synthesis. The only significant sources of vitamin D are fatty fish, animal liver, fish oils, and egg yolks [1].

Vitamin D was initially described at the time of the industrial revolution, when England faced rickets; since then, the relation between vitamin D and calcium-phosphorus metabolism has never been discussed. However, over the last four decades, other activities of vitamin $D$ have been highlighted. Actually, vitamin D is considered as a neurohormone that regulates cell growth and proliferation and immunomodulation.

\section{Vitamin D metabolism}

Exogenous and endogenous vitamin $\mathrm{D}$ is inactive. Two hydroxylation steps, 25 -hydroxylation and $1 \alpha$-hydroxylation, are required to produce the active hormone; 24-hydroxylation is involved in vitamin $\mathrm{D}$ inactivation and catabolism. Cytochrome P450 (CYP) enzymes carry out all hydroxylation steps. Although CYPs are mostly known to carry out oxidation/reduction reactions and liver drug catabolism, they are considered as responsible for vitamin D metabolism [2-4]. This starts from a cutaneous compound, named 7-deidro-cholesterol, which is converted in cholecalciferol by the exposure to ultraviolet B (UVB) radiation. Exposure length and characteristics of the skin can influence the amount of vitamin D production. Classic and novel actions of vitamin D can be summarised according to the tissue where the second hydroxylation takes places.

\section{Liver hydroxylation}

Once cholecalciferol is produced in the skin, it enters the circulation and reaches the liver, where 25-hydroxylation generates 25-OH-cholecalciferol or calcidiol. 25-hydroxylase is physiologically encoded by CYP2R1, but CYP27A1 and CYP3A4 are also involved in cholecalciferol 25-hydroxylation. Once synthesised, calcidiol binds the vitamin D binding protein (DBP) and is secreted into blood, where it

Correspondence: Prof. Marcello Ciaccio, Department of Medical Biotechnologies and Biopathology, University of Palermo,

Via del Vespro 129, 90127 Palermo, Italy, e-mail: marcello.ciaccio@unipa.it

Submitted: 9.03.2018; Accepted: 7.06.2018 
represents the main circulating form of vitamin D. Calcidiol is considered as a reliable marker of vitamin D status.

\section{Kidney hydroxylation}

$1 \alpha$-hydroxylation takes places both in the kidney (renal hydroxylation) and within other tissues and cell types (extra-renal hydroxylation). Kidney $1 \alpha$-hydroxylase originates as a hormone with endocrine effects, responsible for calcium homeostasis (calcaemic actions of vitamin D). Active vitamin $\mathrm{D}$ reaches the gut, where it induces intestinal calcium absorption. Renal $1 \alpha$-hydroxylase is encoded by CYP27B1 and strictly regulated by parathyroid hormone (PTH) [5].

\section{Extra-renal hydroxylation}

Extra-renal tissues having $1 \alpha$-hydroxylases (lung, prostate, brain, immune cells, and placenta) produce a hormone with paracrine and autocrine effects [6]. Extrarenal calcitriol is responsible for non-calcaemic actions of vitamin $\mathrm{D}$, including regulation of cell growth and proliferation and immune system modulation. Macrophages, monocytes, and $\mathrm{T}$ and $\mathrm{B}$ lymphocytes produce active vitamin $\mathrm{D}$, which induces or inhibits immune cell proliferation and cytokine production. Vitamin D expressed by epithelial lung cells has been related to respiratory tract infections, in which its pathogenic role has been strongly suggested, while its role as a biomarker gained less attention, due to the availibility of well-established biomarkers $[7,8]$. Finally, prostate and placenta-vitamin D regulates cells growth and proliferation within such tissues. Extrarenal $1 \alpha$-hydroxylase is stimulated by cytokines and growth factor but not by PTH [9].

\section{Vitamin D receptors}

Most of the roles of vitamin D3 depend on the nuclear vitamin D receptor (VDR). Neo-synthesis of mRNA and protein and cell proliferation take place after vitamin D3 binds VDR. The interaction between VDR and the retinoic acid receptor (RXR) determines the activation of response elements (VDREs) present in the promoter region of several genes. The vitamin D/VDR-RXR-VDREs complex is deemed to regulate about 900 genes [10]. Some nongenomic rapid actions of vitamin D3, such as regulation of adenylate cyclase activity, phospholipase $\mathrm{C}$, and $\mathrm{C}$ kinase, are mediated by the surface receptor $1,25-(\mathrm{OH})$ 2D-MARRS (membrane-associated rapid response steroid binding), also called ERp57/Grp58 [11]. Moreover, a membrane VDR (MVDR), which is similar to the nuclear VDR (NVDR), can mediate other non-genomic rapid actions, such as activation of the phosphatidylinositol 3-kinase and the endothelial nitric oxide synthase [12]. Apart from immune cell types, the prime location of VDR is the brain, hence Vitamin D is defined as a neuro-hormone [13].

\section{Immunomodulation by vitamin $D$}

It has been suggested that vitamin $\mathrm{D}$ action within immune cells resembles that of cytokines, being both a transcription- and a growth factor; as a cytokine, the neurohormone displays pleiotropy, synergy, redundancy, and interaction with surface receptors [14]. Vitamin D plays a pivotal role in promoting innate immune response, by enhancing the production of antimicrobial agents by monocyte and neutrophils, and it is able to modulate the specific immune response. Briefly, the hormone acts by skewing $\mathrm{T}$ cell to Th2 polarisation, attenuating and stimulating Th1 and $\mathrm{Th} 2$ cell proliferation, respectively. Vitamin D can inhibit the synthesis, secretion, and release of Th1 cells anti-inflammatory cytokines (IL-4 and IL-10) whilst inducing those of Th2 cell pro-inflammatory cytokines (IL-1, TNF- $\alpha$, IFN- $\gamma$ ). Furthermore, vitamin D can suppress IL-17 production by Th17 cells and has been proven to promote self-tolerance. Macrophages, monocytes, and B and $\mathrm{T}$ lymphocytes show, even during quiescence, a low expression of VDR, which increases considerably as a result of inflammatory and immunological stimuli [15-19].

\section{Vitamin D and autoimmunity}

Either vitamin D plasma levels or some VDR allelic variants have been largely investigated in immunological disorders, and hypovitaminosis $\mathrm{D}$ is a common finding in several autoimmune diseases [20-24].

VDR polymorphisms (mainly TaqI, FokI, BsmI, and ApaI) and CYP27B1 allelic variants have been investigated in multiple sclerosis (MS), with controversial results. In a case-control study [25] performed in 2016, Abdollahzadeh et al. found a significant difference in ApaI, BsmI, and TaqI but not in FokI allele frequency between the cases and controls. However, Agnello et al. [26] reported no significant difference in allele frequencies and genotype distribution between case and controls when analysing the same VDR polymorphisms. Zhang et al. [27] recently performed a metanalysis on 24 case control studies, including 4013 patients and 4218 healthy controls. The authors found no association between any of the VDR polymorphisms and risk of MS between Asian and Caucasian populations.

A high prevalence of vitamin D deficiency $(<20 \mathrm{ng} / \mathrm{ml})$ has been reported in systemic lupus erythematosus (SLE) [28]. Borba et al. also found an association between hypovitaminosis D and disease activity [29]. However, Amital et al. [30] reported this association to be weak, one year later. When comparing these results, it should be noted that the Amital population included 378 SLE patients while Borba analysed 36 patients. In his recent review [22] on vitamin D in SLE patients, Shoenfeld et al. concluded that vitamin $\mathrm{D}$ deficiency could be a consequence of the disease rather than a cause. Moreover, the authors stated that 
vitamin D supplementation in these patients has very little impact on disease progression.

Controversial findings have been reported by studies evaluating the association between systemic sclerosis and vitamin $\mathrm{D}$ deficiency. A recent review showed that the association between low vitamin D serum levels and skin fibrosis, pulmonary fibrosis, gastrointestinal involvement, and autoantibodies pattern is deeply controversial [21]. However, a recent study [31] performed on 154 patients showed an association between vitamin D deficiency and such clinical features. Because systemic sclerosis is rare, 154 enrolled patients represent a remarkable sample size. Of note, supplementation with oral cholecalciferol was found to be ineffective in increasing $25(\mathrm{OH}) \mathrm{D}$ serum concentrations.

\section{Randomised controlled trials on vitamin D supplementation}

To establish whether there is clinical benefit associated from any treatment, randomised controlled trials (RCTs) are required.

Evidence from RCTs on vitamin D supplementation in MS patients is controversial, as well as in SLE patients.

A few studies performed on MS patients demonstrated that vitamin D supplementation had an effect on the reduction of gadolinium-enhancing lesions on brain magnetic resonance imaging (MRI) [32, 33]. However, previous work found contrasting results, reporting no effect of high-dose supplementation on MRI lesions and clinical outcome [34]. The largest RCT on MS patients was performed by Smolders et al. in 2016 and included 229 subjects [35]. The primary endpoint was defined as the proportion of subjects without evidence of disease activity after 48 weeks. No difference in relapse rate was found between supplementation and placebo groups, but a statistically significant difference in combined unique lesions on MRI (as secondary endpoint) was reported.

Recent evidence from RCTs addressing supplementation in SLE patients demonstrated no impact of vitamin D supplementation on disease activity [36, 37]. In their double-blind, placebo RCT, Aranow et al. analysed 57 SLE patients for the effect of supplementation on interferon (IFN) signature, which is a biomarker of SLE response. The authors concluded that vitamin D supplementation fails to diminish the IFN signature in these patients. Contrasting results were obtained by Lima et al. in the same year; the authors randomised 40 adolescent and young SLE patients to receive oral vitamin $\mathrm{D}$ or placebo. The primary endpoint was defined as disease activity measured by the SLE Disease Activity Index (SLEDAI). The authors found significant improvement in SLEDAI in the vitamin D group compared to placebo, concluding that supplementation is effective in decreasing disease activity.

\section{Conclusions}

There is wide uncertainty on the role of vitamin D in the pathogenesis of autoimmune diseases because it is unclear whether hypovitaminosis D could be a consequence or a cause of autoimmunity. Conflicting evidence on the effect of vitamin D supplementation on these patients emerges from RCT findings. Furthermore, there is a lack of consensus on oral cholecalciferol dosage. More interventional studies are required to better define how vitamin D could represent both a causation agent and a target for therapeutic strategies in autoimmune patients.

The authors declare no conflict of interest.

\section{References}

1. Jones G (2013): Extrarenal vitamin D activation and interactions between vitamin D2, vitamin D3, and vitamin D analogs. Annu Rev Nutr 33: 23-44.

2. Guengerich FP, Waterman MR, Egli M (2016): Recent Structural Insights into Cytochrome P450 Function. Trends Pharmacol Sci 37: 625-640.

3. Caruso A, Bellia C, Pivetti A, et al. (2014): Effects of EPHX1 and CYP3A4 polymorphisms on carbamazepine metabolism in epileptic patients. Pharmgenomics Pers Med 7: 117-120.

4. Jones G, Prosser DE, Kaufmann M (2014): Cytochrome P450-mediated metabolism of Vitamin D. J Lipid Res 55: 13-31.

5. Gil A, Plaza-Diaz J, Mesa AD (2018): Vitamin D: classic and novel action. Ann Nutr Metab 72: 87-95.

6. Hewison M, Adams JS (2011): Extrarenal 1 $\alpha$-hydroxylase. In: Vitamin D ( $3^{\text {rd }}$ ed.), Feldman D, Pike JW, Adams JS (eds.). Academic Press, San Diego, CA: 777-806.

7. Gui B, Chen Q, Hu C, et al. (2017): Effects of calcitriol $(1,25$-dihydroxy-vitamin $\mathrm{D} 3)$ on the inflammatory response induced by H9N2 influenza virus infection in human lung A549 epithelial cells and in mice. Virol $\mathrm{J}$ 14:10.

8. Giulia B, Luisa A, Concetta S, et al. (2015): Procalcitonin and community-acquired pneumonia (CAP) in children. Clin Chim Acta 451: 215-218.

9. Rochel N, Molnár F (2017): Structural aspects of Vitamin D endocrinology. Mol Cell Endocrinol: 22-35.

10. Wang TT, Tavera-Mendoza LE, Laperriere D, et al. (2005): Large-scale in silico and microarray-based identification of direct 1, 25-dihydroxyvitamin D3 target genes. Mol Endocrinol 19: 2685-2695.

11. Hii CS, Ferrante A (2016): The Non-Genomic Actions of Vitamin D. Nutrients 8: 135.

12. Mizwicki MT, Norman AW (2009): The vitamin D sterol-vitamin D receptor ensemble model offers unique insights into both genomic and rapid-response signalling. Sci Signal 2: re4.

13. Cui X, Gooch H, Petty A, et al. (2017): Vitamin D and the brain: Genomic and non-genomic actions. Mol Cell Endocrinol 453: 131-143.

14. Bivona G, Agnello L, Ciaccio M (2017): Vitamin D and Immunomodulation: Is It Time to Change the Reference Values? Ann Clin Lab Sci 47: 508-510. 
15. Vanherwegen AS, Gysemans C, Mathieu C (2017): Regulation of Immune Function by Vitamin D and Its Use in Diseases of Immunity. Endocrinol Metab Clin North Am 46: 1061-1094.

16. Yang CY, Leung PS, Adamopoulos IE, et al. (2013): The implication of vitamin D and autoimmunity: a comprehensive review. Clin Rev Allergy Immunol 45: 217-226.

17. Colotta F, Jansson B, Bonelli F (2017): Modulation of inflammatory and immune responses by vitamin D. J Autoimmun 85 : 78-97.

18. Rosen Y, Daich J, Soliman I, et al. (2016): Vitamin D and autoimmunity. Scand J Rheumatol 45: 439-447.

19. Lin R (2016): Crosstalk between Vitamin D Metabolism, VDR Signalling, and Innate Immunity. Biomed Res Int 2016: 1375858.

20. Agnello L, Scazzone C, Lo Sasso B, et al. (2017): VDBP, CYP27B1, and 25-Hydroxyvitamin D Gene Polymorphism Analyses in a Group of Sicilian Multiple Sclerosis Patients. Biochem Genet 55: 183-192.

21. Bivona G, Agnello L, Pivetti A, et al. (2016): Association between hypovitaminosis D and systemic sclerosis: True or fake? Clin Chim Acta458: 115-119.

22. Shoenfeld Y, Giacomelli R, Azrielant S, et al. (2018): Vitamin D and systemic lupus erythematosus - The hype and the hope. Autoimmun Rev 17: 19-23.

23. Kim D (2017): The Role of Vitamin D in Thyroid Diseases. Int J Mol Sci 18: 1949.

24. Kang SY, Kang JH, Choi JC, et al. (2018): Low serum vitamin D levels in patients with myasthenia gravis. J Clin Neurosci 50: 294-297.

25. Abdollahzadeh R, Fard MS, Rahmani F, et al. (2016): Predisposing role of vitamin $\mathrm{D}$ receptor (VDR) polymorphisms in the development of multiple sclerosis: A case-control study. J Neurol Sci 367: 148-151.

26. Agnello L, Scazzone C, Ragonese P, et al. (2016): Vitamin D receptor polymorphisms and 25-hydroxyvitamin $\mathrm{D}$ in a group of Sicilian multiple sclerosis patients. Neurol Sci 37: 261-267.

27. Zhang YJ, Zhang L, Chen SY, et al. (2018): Association between VDR polymorphisms and multiple sclerosis: systematic review and updated meta-analysis of case-control studies. Neurol Sci 39: 225-234.

28. Kamen DL (2010): Vitamin D in lupus: new kid on the block? Bull Hosp Jt Dis 68: 218.

29. Borba V, Vieira J, Kasamatsu T, et al. (2009): Vitamin D deficiency in patients with active systemic lupus erythematosus. Osteoporis Int 20: 427-433.

30. Amital H, Szekanecz Z, Szücs G, et al. (2010): Serum concentrations of 25-OH vitamin D in patients with systemic lupus erythematosus (SLE) are inversely related to disease activity: is it time to routinely supplement patients with SLE with vitamin D? Ann Rheum Dis 69: 1155-1157.

31. Trombetta AC, Smith V, Gotelli E, et al. (2017): Vitamin $\mathrm{D}$ deficiency and clinical correlations in systemic sclerosis patients: A retrospective analysis for possible future developments. PLoS One 12: e0179062.

32. Kimball SM, Ursell MR, O'Connor P, et al. (2007): Safety of vitamin D3 in adults with multiple sclerosis. Am J Clin Nutr 86: 645-651.

33. Soilu-Hänninen M, Aivo J, Lindström BM, et al. (2012): A randomised, double blind, placebo controlled trial with vitamin D3 as an add on treatment to interferon $\beta-1 b$ in patients with multiple sclerosis. J Neurol Neurosurg Psychiatry 83: 565-571.
34. Stein MS1, Liu Y, Gray OM, et al. (2011): A randomized trial of high-dose vitamin D2 in relapsing-remitting multiple sclerosis. Neurology 77: 1611-1618.

35. Smolders J, Hupperts RMM, Vieth R, et al. (2016): High dose cholecalciferol (vitamin D3) oil as an add-on therapy in subjects with relapsing-remitting multiple sclerosis (RRMS) receiveing subcoutaneous interferon $\beta-1 \mathrm{a}(\operatorname{scIFN} \beta-1 \mathrm{a})$. ECTRIMIS Online Library 147013.

36. Lima GL, Paupitz J, Aikawa NE, et al. (2016): Vitamin D Supplementation in Adolescents and Young Adults With Juvenile Systemic Lupus Erythematosus for Improvement in Disease Activity and Fatigue Scores: A Randomized, Double-Blind, Placebo-Controlled Trial. Arthritis Care Res (Hoboken) 68: 91-98.

37. Aranow C, Kamen DL, Dall'Era M, et al. (2015): Randomized, Double-Blind, Placebo-Controlled Trial of the Effect of Vitamin D3 on the Interferon Signature in Patients With Systemic Lupus Erythematosus. Arthritis Rheumatol 67: 1848-1857. 